\title{
Tikhonov theorem for linear hyperbolic systems
}

\author{
Ying Tang ${ }^{\mathrm{a}}$ Christophe Prieur ${ }^{\mathrm{a}} \quad$ Antoine Girard ${ }^{\mathrm{a}, \mathrm{b}}$ \\ ${ }^{a}$ Gipsa-lab, Grenoble Campus, 11 rue des Mathématiques, BP 46, 38402 Saint Martin d'Hères Cedex, France \\ ${ }^{\mathrm{b}}$ Laboratoire Jean Kuntzmann, Université de Grenoble, BP 53, 38041 Grenoble, France
}

\begin{abstract}
Systems modelled by linear singularly perturbed partial differential equations are considered in this paper. Precisely, a class of linear systems of conservation laws with a small perturbation parameter is introduced. By setting the perturbation parameter to zero, two subsystems, the reduced system standing for the slow dynamics and the boundary-layer system representing the fast dynamics, are computed. It is first proved that the exponential stability of the full system implies the stability of both subsystems. Secondly, a counter example is given to indicate that the converse is not true. Moreover a new Tikhonov theorem for this class of the infinite dimensional systems is stated. The solution of the full system can be approximated by that of the reduced system, and this is proved by Lyapunov techniques. An application to boundary feedback stabilization of gas transport model is used to illustrate the results.
\end{abstract}

Key words: Linear hyperbolic system, Singular perturbation, Tikhonov theorem, Lyapunov function

\section{Introduction}

The early interests in singular perturbation techniques arose from the physical problems exhibiting both fast and slow dynamics, such as DC-motors in Kokotović et al. (1986) where the inductance in the armature circuit plays the role of a perturbation parameter or semiconducting diodes in Smith (1985) where the Debye length is seen as the perturbation parameter. The decomposition of a singularly perturbed system into lower order subsystems, namely the reduced system and the boundary-layer system, provides a powerful tool for stability analysis (e.g. Habets (1974), Chow (1978), Grujic (1981) and Chow and Kokotović (1981)). From late 1980s, singularly perturbed partial differential equations (PDEs) have been considered in research works. This kind of systems describes numerous phenomenon in various fields in physics and engineering, such as fluid dynamics, chemical-reactor, aerodynamics etc. (see Kadalbajoo and Patidar (2003)).

In this paper, we consider a class of linear systems of conservation laws with a small perturbation parameter. The principal motivation for this paper is the model of gas transport through a constant cross section tube represented by Euler equations (see (Winterbone, 2000,

\footnotetext{
$\overline{1}$ E-mail adresses: ying.tang@gipsa-lab.fr, christophe.prieur@gipsa-lab.fr, antoine.girard@imag.fr
}

Chapter 2)). Two time scales for propagation speed exhibit in this model, which can thus be described by a singularly perturbed system of conservation laws.

A first contribution of this paper concerns the stability analysis between a linear singularly perturbed system of conservation laws and its two subsystems. The exponential stability analysis for hyperbolic systems of conservation laws has been considered by many researchers. For instance, a stability criterion for linear hyperbolic systems by characteristics method has been given in Li (1994) and the stability condition considered in Hale and Lunel (1993) relies on the frequency domain. In Coron et al. (2008), a stability condition for the quasilinear systems of conservation laws is introduced by Lyapunov method. In this paper, the first proposition gives a sufficient stability condition for both subsystems. Moreover a counter example is used to illustrate that the exponential stability of the two subsystems does not indicate the stability of the full system. This shows a major difference with what is well known for linear finite dimensional systems (e.g. (Kokotović et al., 1986, Chapter 2)).

A second contribution of our work relates to the approximation of the solution for the singularly perturbed system of conservation laws by the solution for the reduced system. A Tikhonov like theorem is established for the infinite dimensional systems in this paper. Tikhonov theorem describes the limiting behaviour of solutions of the 
perturbed system. It is a powerful tool for analysis of singular perturbation systems. This theorem has been studied in many works for finite dimensional singularly perturbed systems (ODEs) (Verhulst (2007)), (Khalil, 1996, Chapter 9). The approximation of the full system by the reduced subsystem on the finite interval is based on the exponential stability of the boundary-layer subsystem, and that on the infinite interval is achieved by the exponential stability of both subsystems. In the present paper the infinite dimensional case on the infinite interval is stated. This method of approximation can lead the new boundary control strategy for linear hyperbolic systems. For instance, in Tang et al. (2014) boundary control synthesis has been studied based on the singular perturbation method and the slow dynamics is stabilized in finite time.

The paper is organized as follows. The linear singularly perturbed system of conservation laws under consideration is presented in Section 2. The reduced and boundary-layer subsystems are also introduced in this section. In Section 3, we link the exponential stability of the full system of conservation laws with the stability of each of the two subsystems. Section 4 gives a Tikhonov theorem for linear hyperbolic systems of conservation laws. In Section 5, an application to a gas transport model is studied to illustrate the results. Finally, concluding remarks end the paper.

Notation. For a positive integer $n, I_{n}$ is the identity matrix in $\mathbb{R}^{n \times n}$. Given a matrix $A$ in $\mathbb{R}^{n \times n}, A^{-1}$ and $A^{T}$ represent the inverse and the transpose matrix of $A$ respectively. For a symmetric matrix $B$ in $\mathbb{R}^{n \times n}, \lambda_{\min }(B)$ is the minimum eigenvalue of the matrix $B$. The symbol $\star$ in partitioned symmetric matrix stands for the symmetric block. || denotes the usual Euclidean norm in $\mathbb{R}^{n}$ and \|\| is associated with the usual 2-norm of matrices in $\mathbb{R}^{n \times n}$. \|\|$_{L^{2}}$ denotes the associated norm in $L^{2}(0,1)$ space, defined by $\|\xi\|_{L^{2}}=\left(\int_{0}^{1}|\xi|^{2} d x\right)^{\frac{1}{2}}$ for all functions $\xi \in L^{2}(0,1)$. Similarly, the associated norm in $H^{2}(0,1)$ space is denoted by \|\|$_{H^{2}}$, defined for all functions $\psi \in$ $H^{2}(0,1)$, by $\|\psi\|_{H^{2}}=\left(\int_{0}^{1}|\psi|^{2}+\left|\psi_{x}\right|^{2}+\left|\psi_{x x}\right|^{2} d x\right)^{\frac{1}{2}}$. Given a real interval $I$ and a normed space $J, C^{0}(I, J)$ denotes the set of continuous functions from $I$ to $J$.

\section{Linear singularly perturbed systems of con- servation laws}

Firstly, let us consider the following linear singularly perturbed system of conservation laws:

$$
\begin{aligned}
y_{t}(x, t)+\Lambda_{1} y_{x}(x, t) & =0, \\
\epsilon z_{t}(x, t)+\Lambda_{2} z_{x}(x, t) & =0,
\end{aligned}
$$

where $x \in[0,1], t \in[0,+\infty), y:[0,1] \times[0,+\infty) \rightarrow \mathbb{R}^{n}$, $z:[0,1] \times[0,+\infty) \rightarrow \mathbb{R}^{m}, \Lambda_{1}$ is a diagonal positive matrix in $\mathbb{R}^{n \times n}, \Lambda_{2}$ is a diagonal positive matrix in $\mathbb{R}^{m \times m}$, the perturbation parameter $\epsilon$ is a small positive value. Moreover, we consider the following boundary conditions:

$$
\left(\begin{array}{l}
y(0, t) \\
z(0, t)
\end{array}\right)=G\left(\begin{array}{l}
y(1, t) \\
z(1, t)
\end{array}\right), t \in[0,+\infty)
$$

where $G=\left(\begin{array}{ll}G_{11} & G_{12} \\ G_{21} & G_{22}\end{array}\right)$ is a constant matrix in $\mathbb{R}^{(n+m) \times(n+m)}$ with the matrices $G_{11}$ in $\mathbb{R}^{n \times n}, G_{12}$ in $\mathbb{R}^{n \times m}, G_{21}$ in $\mathbb{R}^{m \times n}$ and $G_{22}$ in $\mathbb{R}^{m \times m}$.

Given two functions $y^{0}:[0,1] \rightarrow \mathbb{R}^{n}$ and $z^{0}:[0,1] \rightarrow$ $\mathbb{R}^{m}$, the initial conditions are:

$$
\left(\begin{array}{l}
y(x, 0) \\
z(x, 0)
\end{array}\right)=\left(\begin{array}{l}
y^{0}(x) \\
z^{0}(x)
\end{array}\right), x \in[0,1]
$$

Remark 1 Let us recall the existence of the solutions to the Cauchy problem (1)-(3). According to Section 2.1 in Coron (2007), for all $\left(\begin{array}{c}y^{0} \\ z^{0}\end{array}\right) \in L^{2}(0,1)$, there exists a unique solution $\left(\begin{array}{l}y \\ z\end{array}\right) \in C^{0}\left([0,+\infty), L^{2}(0,1)\right)$ for the Cauchy problem (1)-(3). By Proposition 2.1 in Coron et al. (2008), for every $\left(\begin{array}{l}y^{0} \\ z^{0}\end{array}\right) \in H^{2}(0,1)$ satisfying the following compatibility conditions:

$$
\begin{aligned}
\left(\begin{array}{l}
y^{0}(0) \\
z^{0}(0)
\end{array}\right) & =G\left(\begin{array}{c}
y^{0}(1) \\
z^{0}(1)
\end{array}\right), \\
\left(\begin{array}{c}
\Lambda_{1} y_{x}^{0}(0) \\
\epsilon^{-1} \Lambda_{2} z_{x}^{0}(0)
\end{array}\right) & =G\left(\begin{array}{c}
\Lambda_{1} y_{x}^{0}(1) \\
\epsilon^{-1} \Lambda_{2} z_{x}^{0}(1)
\end{array}\right),
\end{aligned}
$$

the Cauchy problem (1)-(3) has a unique maximal classical solution $\left(\begin{array}{l}y \\ z\end{array}\right) \in C^{0}\left([0,+\infty), H^{2}(0,1)\right)$.

Remark 2 In Perrollaz and Rosier (2014), a $2 \times 2$ quasilinear hyperbolic system is considered in $C^{0}([0, T] \times$ $\left.[0,1] ; \mathbb{R}^{2}\right)$ instead of $H^{2}(0,1)$, avoiding so strong compatibility conditions. However, in the following of the present paper, the $\mathrm{H}^{2}$ convergence of a system is mandatory thus the compatibility conditions should be considered.

Considering infinite dimensional systems (PDEs), let us compute the two subsystems for (1)-(2), the reduced and the boundary-layer systems. Inspired by the approach for finite dimensional systems (ODEs) in (Saberi and 
Khalil (1984)) and (Khalil, 1996, Chapter 9), the two subsystems are formally calculated as follows. Setting $\epsilon=0$ in system (1) yields

$$
\begin{aligned}
y_{t}(x, t)+\Lambda_{1} y_{x}(x, t) & =0, \\
z_{x}(x, t) & =0 .
\end{aligned}
$$

Substituting (6b) into the boundary conditions (2) and assuming $\left(I_{m}-G_{22}\right)$ invertible yields

$$
\begin{aligned}
y(0, t) & =\left(G_{11}+G_{12}\left(I_{m}-G_{22}\right)^{-1} G_{21}\right) y(1, t), \\
z(., t) & =\left(I_{m}-G_{22}\right)^{-1} G_{21} y(1, t) .
\end{aligned}
$$

Then, the reduced system is defined as

$$
\bar{y}_{t}(x, t)+\Lambda_{1} \bar{y}_{x}(x, t)=0, x \in[0,1], t \in[0,+\infty),
$$

with the boundary condition

$$
\bar{y}(0, t)=G_{r} \bar{y}(1, t), t \in[0,+\infty),
$$

where $G_{r}=G_{11}+G_{12}\left(I_{m}-G_{22}\right)^{-1} G_{21}$, whereas the initial condition is given as

$$
\bar{y}(x, 0)=\bar{y}^{0}(x)=y^{0}(x), x \in[0,1] .
$$

To define the boundary-layer system, let first perform the change of variable

$$
\bar{z}(x, t)=z(x, t)-\left(I_{m}-G_{22}\right)^{-1} G_{21} y(1, t) .
$$

This shifts the equilibrium of $z$ to the origin. Let us use a new time variable $\tau=\frac{t}{\epsilon}$. In the $\tau$ time scale, $y(1, t)$ in (11) is considered as a fixed parameter with respect to time. Then, the boundary-layer system is defined as

$$
\bar{z}_{\tau}(x, \tau)+\Lambda_{2} \bar{z}_{x}(x, \tau)=0, x \in[0,1], \tau \in[0,+\infty),
$$

with the boundary condition

$$
\bar{z}(0, \tau)=G_{22} \bar{z}(1, \tau), \tau \in[0,+\infty),
$$

whereas the initial condition is given as

$\bar{z}(x, 0)=\bar{z}^{0}(x)=z^{0}(x)-\left(I_{m}-G_{22}\right)^{-1} G_{21} y^{0}(1), x \in[0,1]$.

\section{Stability of reduced and boundary-layer sys- tems}

In this section, our aim is to show how the stability of the singularly perturbed system of conservation laws (1)(2) is related to the stability of the two subsystems, the reduced system (8)-(9) and the boundary-layer system (12)-(13)

Let us recall the following definition introduced in Coron et al. (2008):
Definition 1 For all matrices $G \in \mathbb{R}^{(n+m) \times(n+m)}$,

$$
\rho_{1}(G)=\inf \left\{\left\|\Delta G \Delta^{-1}\right\|, \Delta \in D_{(n+m),+}\right\},
$$

where $D_{(n+m),+}$ denotes the set of diagonal positive matrix in $\mathbb{R}^{(n+m) \times(n+m)}$.

The following definition is adopted for the exponential stability of the linear singularly perturbed system of conservation laws (1)-(2) in $L^{2}$-norm.

Definition 2 The linear system of conservation laws (1)-(2) is exponentially stable to the origin in $L^{2}$-norm if there exist $\gamma_{1}>0$ and $C_{1}>0$, such that for every $\left(\begin{array}{l}y^{0} \\ z^{0}\end{array}\right) \in L^{2}(0,1)$, the solution to the system (1)-(2) satisfies

$$
\left\|\left(\begin{array}{l}
y(., t) \\
z(., t)
\end{array}\right)\right\|_{L^{2}} \leqslant C_{1} e^{-\gamma_{1} t}\left\|\left(\begin{array}{l}
y^{0} \\
z^{0}
\end{array}\right)\right\|_{L^{2}}, t \in[0,+\infty) .
$$

Similarly the exponential stability of the linear system of conservation laws (1)-(2) in $H^{2}$-norm is defined by the following definition.

Definition 3 The linear system of conservation laws (1)-(2) is exponentially stable to the origin in $\mathrm{H}^{2}$-norm if there exist $\gamma_{2}>0$ and $C_{2}>0$, such that for every $\left(\begin{array}{c}y^{0} \\ z^{0}\end{array}\right) \in H^{2}(0,1)$ satisfying the compatibility conditions (4)-(5), the solution to the system (1)-(2) satisfies

$$
\left\|\left(\begin{array}{l}
y(., t) \\
z(., t)
\end{array}\right)\right\|_{H^{2}} \leqslant C_{2} e^{-\gamma_{2} t}\left\|\left(\begin{array}{c}
y^{0} \\
z^{0}
\end{array}\right)\right\|_{H^{2}}, t \in[0,+\infty) .
$$

Similarly, we can define the exponential stability in $L^{2}$ norm and $H^{2}$-norm for the reduced and boundary-layer systems.

Let us recall the following result for linear hyperbolic systems:

Theorem 1 (Coron et al. (2008), Diagne et al. (2012)) If $\rho_{1}(G)<1$ (resp. $\rho_{1}\left(G_{r}\right)<1, \rho_{1}\left(G_{22}\right)<1$ ), then the linear system (1)-(2) (resp. the reduced system (8)-(9), the boundary-layer system (12)-(13)) is exponentially stable to the origin in $L^{2}$-norm and $H^{2}$-norm.

With the above theorem, we are ready to give a proposition which is about the stability of the reduced and the boundary-layer systems.

Proposition 1 If $\rho_{1}(G)<1$, then the reduced system (8)-(9) and the boundary-layer system (12)-(13) are exponentially stable to the origin in $L^{2}$-norm and $H^{2}$-norm. 
Proof. Let first prove the stability of the reduced system (8)-(9). Let $\rho_{1}(G)<\alpha^{*}<1$ and $\Delta \in D_{(n+m),+}$ such that $\left\|\Delta G \Delta^{-1}\right\| \leqslant \alpha^{*}$. Let $\Delta_{1} \in D_{n,+}$ and $\Delta_{2} \in D_{m,+}$, $\Delta$ be such that

$$
\Delta=\left(\begin{array}{cc}
\Delta_{1} & 0 \\
0 & \Delta_{2}
\end{array}\right)
$$

Consider

$$
\widetilde{Y}=\left(\begin{array}{c}
Y \\
\Delta_{2}\left(I_{m}-G_{22}\right)^{-1} G_{21} \Delta_{1}^{-1} Y
\end{array}\right), \tilde{Y} \in \mathbb{R}^{n+m},
$$

where $Y$ is an arbitrary vector of $\mathbb{R}^{n}$. It follows directly

$\Delta G \Delta^{-1} \widetilde{Y}=\left(\begin{array}{c}\Delta_{1}\left(G_{11}+G_{12}\left(I_{m}-G_{22}\right)^{-1} G_{21}\right) \Delta_{1}^{-1} Y \\ \Delta_{2}\left(I_{m}-G_{22}\right)^{-1} G_{21} \Delta_{1}^{-1} Y\end{array}\right)$.

Since $\left\|\Delta G \Delta^{-1}\right\| \leqslant \alpha^{*}$, and it follows from (16)

$$
\left|\Delta G \Delta^{-1} \widetilde{Y}\right| \leqslant \alpha^{*}|\widetilde{Y}|
$$

hence

$$
\left|\Delta_{1}\left(G_{11}+G_{12}\left(I_{m}-G_{22}\right)^{-1} G_{21}\right) \Delta_{1}^{-1} Y\right| \leqslant \alpha^{*}|Y| .
$$

The previous inequality holds for all $Y \in \mathbb{R}^{n}$, therefore

$$
\left\|\Delta_{1}\left(G_{11}+G_{12}\left(I_{m}-G_{22}\right)^{-1} G_{21}\right) \Delta_{1}^{-1}\right\| \leqslant \alpha^{*}<1,
$$

which implies $\rho_{1}\left(G_{r}\right)<1$. Therefore, with Theorem 1, the reduced system (8)-(9) is exponentially stable in $L^{2}$ norm and $H^{2}$-norm.

Next let prove the stability of the boundary-layer system (12)-(13). Similarly, consider

$$
\widetilde{Z}=\left(\begin{array}{l}
0 \\
Z
\end{array}\right), \quad \widetilde{Z} \in \mathbb{R}^{n+m},
$$

where $Z$ is an arbitrary vector of $\mathbb{R}^{m}$. It follows directly

$$
\Delta G \Delta^{-1} \widetilde{Z}=\left(\begin{array}{c}
\Delta_{1} G_{12} \Delta_{2}^{-1} Z \\
\Delta_{2} G_{22} \Delta_{2}^{-1} Z
\end{array}\right)
$$

Since $\left\|\Delta G \Delta^{-1}\right\| \leqslant \alpha^{*},(18)$ follows

$$
\left|\Delta G \Delta^{-1} \widetilde{Z}\right| \leqslant \alpha^{*}|\widetilde{Z}|
$$

hence

$$
\left|\Delta_{2} G_{22} \Delta_{2}^{-1} Z\right| \leqslant \alpha^{*}|Z| .
$$

The previous inequality holds for all $Z \in \mathbb{R}^{m}$, therefore

$$
\left\|\Delta_{2} G_{22} \Delta_{2}^{-1}\right\| \leqslant \alpha^{*}<1,
$$

which implies $\rho_{1}\left(G_{22}\right)<1$. Therefore, with Theorem 1 , the boundary-layer system (12)-(13) is exponentially stable in $L^{2}$-norm and $H^{2}$-norm. This concludes the proof of Proposition 1.

The stability criterion $\rho_{1}(G)<1$ is a sufficient condition for stability of the reduced system (8)-(9) and the boundary-layer system (12)-(13). On the other hand, the stability of the two subsystems does not guarantee the stability of the overall system (1)-(2). To see this, let us consider the following example. Let $\Lambda_{1}=\Lambda_{2}=1$ in (1) with $n=m=1$. The boundary condition of the singularly perturbed system in (2) is chosen as $G_{11}=2.5$, $G_{12}=-1, G_{21}=1, G_{22}=0.5$. The boundary condition of the reduced system in (9) is computed as $G_{r}=0.5$. It holds $\rho_{1}\left(G_{r}\right)<1$. By Theorem 1 the reduced system (8)-(9) is exponentially stable in $L^{2}$-norm and $H^{2}$ norm. The boundary condition of the boundary-layer system in (13) is $G_{22}=0.5$. It holds $\rho_{1}\left(G_{22}\right)<1$. The boundary-layer system (12)-(13) is exponentially stable in $L^{2}$-norm and $H^{2}$-norm according to Theorem 1 . Now let us check the stability condition $\rho_{1}(G)<1$, which is equivalent to find a diagonal positive matrix $\Delta$ such that $\left\|\Delta G \Delta^{-1}\right\|<1$ and it is in fact equivalent to (see Coron et al. (2008) section 4)

$$
G^{T} \Delta^{2} G<\Delta^{2} .
$$

There is no loss of generality to look for $\Delta=\left(\begin{array}{ll}1 & 0 \\ 0 & b\end{array}\right)$. Straightforward computations show that there is no such matrix $\Delta$ which satisfies the condition (20), thus $\rho_{1}(G) \geqslant 1$. Note that, Proposition 3.7 in Coron et al. (2008) implies that $\rho_{1}(G)<1$ is a necessary and sufficient condition for stability of linear hyperbolic systems with dimension 1 to 5 . As this example is a linear singularly perturbed system of two conservation laws, it is not exponentially stable neither in $L^{2}$-norm nor in $H^{2}$-norm, although the reduced and boundary-layer systems are both exponentially stable.

See also Tang et al. (2013) where under the exponential stability of the both subsystems, an additional condition is introduced for the exponential stability of the linear singularly perturbed system.

\section{Approximation theorem for linear singularly perturbed system of conservation laws}

A Tikhonov like theorem is given in this section. It presents how solutions to the linear singularly perturbed system of conservation laws (1)-(2) can be approximated by solutions to the reduced system (8)-(9). It is based on the stability condition we considered in the last section.

Theorem 2 Consider the linear singularly perturbed system of conservation laws (1)-(2). Assume that the boundary conditions $G$ satisfy $\rho_{1}(G)<1$, then, for all 
initial conditions $y^{0} \in H^{2}(0,1)$ satisfying the compatibility conditions $y^{0}(0)=G_{r} y^{0}(1), \Lambda_{1} y_{x}^{0}(0)=G_{r} \Lambda_{1} y_{x}^{0}(1)$, and $z \in L^{2}(0,1)$, there exists positive values $\epsilon^{*}, C, C^{\prime}$ and $\omega$ such that for all $0<\epsilon<\epsilon^{*}$ and for all $t \geqslant 0$,

$$
\begin{gathered}
\|y(., t)-\bar{y}(., t)\|_{L^{2}}^{2} \leqslant C \epsilon e^{-\omega t} \\
\int_{0}^{\infty}\left\|z(., t)-\left(I-G_{22}\right)^{-1} G_{21} \bar{y}(1, t)\right\|_{L^{2}}^{2} d t \leqslant C^{\prime} \epsilon
\end{gathered}
$$

Before stating the proof of Theorem 2, let us first perform the following change of variables

$$
\begin{aligned}
& \eta(x, t)=y(x, t)-\bar{y}(x, t) \\
& \delta(x, t)=z(x, t)-\left(I_{m}-G_{22}\right)^{-1} G_{21} \bar{y}(1, t),
\end{aligned}
$$

where $\eta$ is the error between $y$ in the full system and $\bar{y}$ in the reduced system, $\delta$ is the error between $z$ in the full system and its equilibrium point $\left(I_{m}-G_{22}\right)^{-1} G_{21} \bar{y}(1, t)$. In the new variables $(\eta, \delta)$, the system is

$$
\begin{aligned}
\eta_{t}+\Lambda_{1} \eta_{x} & =0 \\
\epsilon \delta_{t}+\Lambda_{2} \delta_{x} & =\epsilon\left(I_{m}-G_{22}\right)^{-1} G_{21} \Lambda_{1} \bar{y}_{x}(1, t) .
\end{aligned}
$$

The boundary conditions are calculated as follows

$$
\begin{aligned}
\eta(0, t)= & y(0, t)-\bar{y}(0, t) \\
= & G_{11} y(1, t)+G_{12} z(1, t)-G_{r} \bar{y}(1, t) \\
= & G_{11}(y(1, t)-\bar{y}(1, t)) \\
& +G_{12}\left(z(1, t)-\left(I_{m}-G_{22}\right)^{-1} G_{21} \bar{y}(1, t)\right) \\
= & G_{11} \eta(1, t)+G_{12} \delta(1, t),
\end{aligned}
$$

and

$$
\begin{aligned}
\delta(0, t)= & z(0, t)-\left(I_{m}-G_{22}\right)^{-1} G_{21} \bar{y}(1, t) \\
= & G_{21} y(1, t)+G_{22} z(1, t)-\left(I_{m}-G_{22}\right)^{-1} G_{21} \bar{y}(1, t) \\
= & G_{21}(y(1, t)-\bar{y}(1, t)) \\
& +G_{22} z(1, t)+\left(\left(I_{m}-\left(I_{m}-G_{22}\right)^{-1}\right) G_{21} \bar{y}(1, t)\right) \\
= & G_{21} \eta(1, t)+G_{22} \delta(1, t) .
\end{aligned}
$$

We summarize the boundary conditions for system (23) as follows

$$
\left(\begin{array}{l}
\eta(0, t) \\
\delta(0, t)
\end{array}\right)=G\left(\begin{array}{l}
\eta(1, t) \\
\delta(1, t)
\end{array}\right)
$$

Let consider the following Lyapunov function candidate for system (23)-(24)

$$
V_{\epsilon}(\eta, \delta)=\int_{0}^{1} e^{-\mu x}\left(\eta^{T} Q \eta+\epsilon \delta^{T} P \delta\right) d x
$$

with $\mu>0, Q$ a diagonal positive matrix in $\mathbb{R}^{n \times n}$ and $P$ a diagonal positive matrix in $\mathbb{R}^{m \times m}$.

The time derivative of $V_{\epsilon}(\eta, \delta)$ is estimated in Lemma 1.

Lemma 1 If the boundary conditions satisfy $\rho_{1}(G)<1$, then there exist a positive value $\mu$ and diagonal positive matrices $P$ and $Q$ such that for all $\epsilon>0$ and $\kappa>0$, it holds

$\dot{V}_{\epsilon}(\eta, \delta) \leqslant-\mu \int_{0}^{1} e^{-\mu x} \eta^{T} Q \Lambda_{1} \eta d x$

$-\left(\mu-\frac{\epsilon \kappa\left\|P\left(I_{m}-G_{22}\right)^{-1} G_{21} \Lambda_{1}\right\|}{\lambda_{\min }\left(P \Lambda_{2}\right)}\right) \int_{0}^{1} e^{-\mu x} \delta^{T} P \Lambda_{2} \delta d x$

$+\frac{\epsilon\left\|P\left(I_{m}-G_{22}\right)^{-1} G_{21} \Lambda_{1}\right\|}{\kappa}\left|\bar{y}_{x}(1, t)\right|^{2}$.

Proof. Computing the time derivative of $V_{\epsilon}(\eta, \delta)$ along (23) and integrating by parts yield (see Diagne et al. (2012))

$$
\dot{V}_{\epsilon}(\eta, \delta)=N_{1}+N_{2}+N_{3}
$$

with:

$$
\begin{aligned}
& N_{1}=-\left(e^{-\mu x} \eta^{T} Q \Lambda_{1} \eta\right)_{x=0}^{x=1}-\left(e^{-\mu x} \delta^{T} P \Lambda_{2} \delta\right)_{x=0}^{x=1} \\
& N_{2}=-\mu \int_{0}^{1} e^{-\mu x}\left(\eta^{T} Q \Lambda_{1} \eta+\delta^{T} P \Lambda_{2} \delta\right) d x \\
& N_{3}=2 \epsilon \int_{0}^{1} e^{-\mu x} \delta^{T} P\left(I_{m}-G_{22}\right)^{-1} G_{21} \Lambda_{1} \bar{y}_{x}(1, t) d x
\end{aligned}
$$

Using boundary conditions (24), the first term $N_{1}$ follows

$$
\begin{aligned}
N_{1}= & -\left(e^{-\mu} \eta^{T}(1) Q \Lambda_{1} \eta(1)\right. \\
& \left.-\left(G_{11} \eta(1)+G_{12} \delta(1)\right)^{T} Q \Lambda_{1}\left(G_{11} \eta(1)+G_{12} \delta(1)\right)\right) \\
& -\left(e^{-\mu} \delta^{T}(1) P \Lambda_{2} \delta(1)\right. \\
& \left.-\left(G_{21} \eta(1)+G_{22} \delta(1)\right)^{T} P \Lambda_{2}\left(G_{21} \eta(1)+G_{22} \delta(1)\right)\right) .
\end{aligned}
$$

Developing and reorganizing (31), we obtain

$$
\begin{array}{r}
N_{1}=-\left(\begin{array}{c}
\eta(1) \\
\delta(1)
\end{array}\right)^{T}\left(\begin{array}{cc}
e^{-\mu} Q \Lambda_{1} & 0 \\
0 & e^{-\mu} P \Lambda_{2}
\end{array}\right)\left(\begin{array}{c}
\eta(1) \\
\delta(1)
\end{array}\right) \\
+\left(\begin{array}{c}
\eta(1) \\
\delta(1)
\end{array}\right)^{T}\left(\begin{array}{c}
G_{11}^{T} Q \Lambda_{1} G_{11}+G_{21}^{T} P \Lambda_{2} G_{21} \\
\star \\
G_{11}^{T} Q \Lambda_{1} G_{12}+G_{21}^{T} P \Lambda_{2} G_{22} \\
G_{22}^{T} P \Lambda_{2} G_{22}+G_{12}^{T} Q \Lambda_{1} G_{12}
\end{array}\right)\left(\begin{array}{c}
\eta(1) \\
\delta(1)
\end{array}\right) .
\end{array}
$$


Since $\rho_{1}(G)<1$, let $\Delta=\left(\begin{array}{cc}\Delta_{1} & 0 \\ 0 & \Delta_{2}\end{array}\right)$, such that $\left\|\Delta G \Delta^{-1}\right\|=\sigma^{*}<1$, there exists $0<\mu \leqslant-2 \ln \sigma^{*}$. Choosing matrices $Q$ and $P$ such that $Q=\Delta_{1}^{2} \Lambda_{1}^{-1}$, $P=\Delta_{2}^{2} \Lambda_{2}^{-1}$, it follows

$N_{1} \leqslant-\left(\begin{array}{c}\eta(1) \\ \delta(1)\end{array}\right)^{T}\left(e^{-\mu} \Delta^{2}-\sigma^{* 2} \Delta^{2}\right)\left(\begin{array}{l}\eta(1) \\ \delta(1)\end{array}\right) \leqslant 0$.

The second term $N_{2}$ is always non positive.

According to Cauchy-Schwarz inequality, the third term $N_{3}$ is bounded as follows

$N_{3} \leqslant 2 \epsilon\left\|P\left(I_{m}-G_{22}\right)^{-1} G_{21} \Lambda_{1}\right\| \int_{0}^{1} e^{-\mu x}|\delta|\left|\bar{y}_{x}(1, t)\right| d x$.

By Young's inequality, for all $\kappa>0$, it holds

$$
\begin{aligned}
N_{3} \leqslant & \epsilon \kappa\left\|P\left(I_{m}-G_{22}\right)^{-1} G_{21} \Lambda_{1}\right\| \int_{0}^{1} e^{-\mu x}|\delta|^{2} d x \\
& +\frac{\epsilon\left\|P\left(I_{m}-G_{22}\right)^{-1} G_{21} \Lambda_{1}\right\|}{\kappa} \int_{0}^{1} e^{-\mu x}\left|\bar{y}_{x}(1, t)\right|^{2} d x .
\end{aligned}
$$

Combining (29), (33) and (34), we obtain

$\dot{V}_{\epsilon}(\eta, \delta) \leqslant-\mu \int_{0}^{1} e^{-\mu x} \eta^{T} Q \Lambda_{1} \eta d x$

$-\left(\mu-\frac{\epsilon \kappa\left\|P\left(I_{m}-G_{22}\right)^{-1} G_{21} \Lambda_{1}\right\|}{\lambda_{\min }\left(P \Lambda_{2}\right)}\right) \int_{0}^{1} e^{-\mu x} \delta^{T} P \Lambda_{2} \delta d x$

$+\frac{\epsilon\left\|P\left(I_{m}-G_{22}\right)^{-1} G_{21} \Lambda_{1}\right\|}{\kappa} \int_{0}^{1} e^{-\mu x}\left|\bar{y}_{x}(1, t)\right|^{2} d x$.

This concludes the proof of Lemma 1.

By Poincaré inequality $\left|\bar{y}_{x}(1, t)\right|$ is bounded as follows

$$
\begin{aligned}
\left|\bar{y}_{x}(1, t)\right| & =\left|\int_{0}^{1}\left(x \bar{y}_{x x}+\bar{y}_{x}\right) d x\right| \\
& \leqslant \int_{0}^{1}\left(\left|\bar{y}_{x x}\right|+\left|\bar{y}_{x}\right|+|\bar{y}|\right) d x \\
& \leqslant \sqrt{3}\|\bar{y}(., t)\|_{H^{2}} .
\end{aligned}
$$

Thus, in order to bound the term $\left|\bar{y}_{x}(1, t)\right|$, it is necessary to analyze the stability of the reduced system in $\mathrm{H}^{2}$ norm.

Since we choose the initial condition of the reduced system $\bar{y}^{0}=y^{0}$, thus $\bar{y}^{0} \in H^{2}(0,1)$ and the compatibility conditions $\bar{y}^{0}(0)=G_{r} \bar{y}^{0}(1)$ and $\Lambda_{1} \bar{y}_{x}^{0}(0)=G_{r} \Lambda_{1} \bar{y}_{x}^{0}(1)$ are satisfied. The estimate of $\|\bar{y}(., t)\|_{H^{2}}^{2}$ is given in the following Lemma 2.

Lemma 2 If $\rho_{1}(G)<1$, let $\mu$ as in Lemma 1, there exists a positive value $C_{0}$ such that for any initial condition $\bar{y}^{0} \in H^{2}(0,1)$ satisfying the compatibility conditions $\bar{y}^{0}(0)=G_{r} \bar{y}^{0}(1)$ and $\Lambda_{1} \bar{y}_{x}^{0}(0)=G_{r} \Lambda_{1} \bar{y}_{x}^{0}(1)$, it holds

$$
\|\bar{y}(., t)\|_{H^{2}}^{2} \leqslant C_{0} e^{-\mu \lambda_{\min }\left(\Lambda_{1}\right) t}\left\|\bar{y}^{0}\right\|_{H^{2}}^{2}, \quad t \geqslant 0 .
$$

Proof. $\bar{y}^{0} \in H^{2}(0,1)$ and the compatibility conditions imply that $\bar{y}(., t) \in H^{2}(0,1)$ for all $t \geqslant 0$. Therefore we consider the following Lyapunov function candidate for the reduced system (8)-(9)

$$
V_{1}(\bar{y})=\int_{0}^{1} e^{-\mu x}\left(\bar{y}^{T} \bar{Q}_{1} \bar{y}+\bar{y}_{x}^{T} \bar{Q}_{2} \bar{y}_{x}+\bar{y}_{x x}^{T} \bar{Q}_{3} \bar{y}_{x x}\right) d x
$$

where $\bar{Q}_{1}, \bar{Q}_{2}, \bar{Q}_{3}$ are diagonal positive matrices in $\mathbb{R}^{n \times n}$. We rewrite $V_{1}(\bar{y})$ as

$$
V_{1}(\bar{y})=V_{10}+V_{11}+V_{12},
$$

with

$$
\begin{gathered}
V_{10}=\int_{0}^{1} e^{-\mu x} \bar{y}^{T} \bar{Q}_{1} \bar{y} d x, \\
V_{11}=\int_{0}^{1} e^{-\mu x} \bar{y}_{x}^{T} \bar{Q}_{2} \bar{y}_{x} d x, \\
V_{12}=\int_{0}^{1} e^{-\mu x} \bar{y}_{x x}^{T} \bar{Q}_{3} \bar{y}_{x x} d x .
\end{gathered}
$$

Computing the time derivative of $V_{10}$ along (8) and integrating by parts we have

$$
\dot{V}_{10}=-\left[e^{-\mu x} \bar{y}^{T} \bar{Q}_{1} \Lambda_{1} \bar{y}\right]_{x=0}^{x=1}-\mu \int_{0}^{1} e^{-\mu x} \bar{y}^{T} \bar{Q}_{1} \Lambda_{1} \bar{y} d x .
$$

Using the boundary condition (9), it follows from (38)

$$
\begin{aligned}
\dot{V}_{10}= & -\left(e^{-\mu} \bar{y}^{T}(1) \bar{Q}_{1} \Lambda_{1} \bar{y}(1)-\left(G_{r} \bar{y}(1)\right)^{T} \bar{Q}_{1} \Lambda_{1}\left(G_{r} \bar{y}(1)\right)\right) \\
& -\mu \int_{0}^{1} e^{-\mu x} \bar{y}^{T} \bar{Q}_{1} \Lambda_{1} \bar{y} d x
\end{aligned}
$$

Developing and reorganizing (39) we get

$$
\begin{aligned}
\dot{V}_{10}= & -\bar{y}^{T}(1)\left(e^{-\mu} \bar{Q}_{1} \Lambda_{1}-G_{r}^{T} \bar{Q}_{1} \Lambda_{1} G_{r}\right) \bar{y}(1) \\
& -\mu \int_{0}^{1} e^{-\mu x} \bar{y}^{T} \bar{Q}_{1} \Lambda_{1} \bar{y} d x
\end{aligned}
$$


Similar to the proof of Proposition 1, we show that $\left\|\Delta G \Delta^{-1}\right\|<1$ implies $\left\|\Delta_{1} G_{r} \Delta_{1}^{-1}\right\|<1$ which is equivalent to $\Delta_{1}^{2}-G_{r}^{T} \Delta_{1}^{2} G_{r}>0$. By selecting $\bar{Q}_{1}=\Delta_{1}^{2} \Lambda_{1}^{-1}$, it is deduced from (40)

$$
\dot{V}_{10} \leqslant-\mu \int_{0}^{1} e^{-\mu x} \bar{y}^{T} \bar{Q}_{1} \Lambda_{1} \bar{y} d x .
$$

Differentiating system (8) with respect to $x$ yields

$$
\bar{y}_{x t}+\Lambda_{1} \bar{y}_{x x}=0,
$$

differentiating (9) with respect to $t$ and using (8), the boundary condition is calculated as

$$
\bar{y}_{x}(0, t)=\Lambda_{1}^{-1} G_{r} \Lambda_{1} \bar{y}_{x}(1, t) .
$$

Computing the time derivative of $V_{11}$ along (42) and integrating by parts we have

$\dot{V}_{11}=-\left[e^{-\mu x} \bar{y}_{x}^{T} \bar{Q}_{2} \Lambda_{1} \bar{y}_{x}\right]_{x=0}^{x=1}-\mu \int_{0}^{1} e^{-\mu x} \bar{y}_{x}^{T} \bar{Q}_{2} \Lambda_{1} \bar{y}_{x} d x$.

Using the boundary condition (43), it follows from (44)

$$
\begin{aligned}
\dot{V}_{11}= & -\left(e^{-\mu} \bar{y}_{x}^{T}(1) \bar{Q}_{2} \Lambda_{1} \bar{y}_{x}(1)\right. \\
& \left.-\left(\Lambda_{1}^{-1} G_{r} \Lambda_{1} \bar{y}_{x}(1)\right)^{T} \bar{Q}_{2} \Lambda_{1}\left(\Lambda_{1}^{-1} G_{r} \Lambda_{1} \bar{y}_{x}(1)\right)\right) \\
& -\mu \int_{0}^{1} e^{-\mu x} \bar{y}_{x}^{T} \bar{Q}_{2} \Lambda_{1} \bar{y}_{x} d x .
\end{aligned}
$$

Developing and reorganizing (45) we get

$$
\begin{aligned}
\dot{V}_{11}= & -\bar{y}_{x}^{T}(1) \Lambda_{1}\left(e^{-\mu} \Lambda_{1}^{-1} \bar{Q}_{2}-G_{r}^{T} \Lambda_{1}^{-1} \bar{Q}_{2} G_{r}\right) \Lambda_{1} \bar{y}_{x}(1) \\
& -\mu \int_{0}^{1} e^{-\mu x} \bar{y}_{x}^{T} \bar{Q}_{2} \Lambda_{1} \bar{y}_{x} d x .
\end{aligned}
$$

Similarly, by selecting $\bar{Q}_{2}=\Lambda_{1} \Delta_{1}^{2}$, it is deduced from (46)

$$
\dot{V}_{11} \leqslant-\mu \int_{0}^{1} e^{-\mu x} \bar{y}_{x}^{T} \bar{Q}_{2} \Lambda_{1} \bar{y}_{x} d x .
$$

Differentiating system (42) with respect to $x$ yields

$$
\bar{y}_{x x t}+\Lambda_{1} \bar{y}_{x x x}=0,
$$

differentiating (43) with respect to $t$ and using (42), the boundary condition is calculated as

$$
\bar{y}_{x x}(0, t)=\Lambda_{1}^{-2} G_{r} \Lambda_{1}^{2} \bar{y}_{x x}(1, t) .
$$

Computing the time derivative of $V_{12}$ along (48) and integrating by parts we have

$$
\begin{aligned}
\dot{V}_{12}= & \left.-\left[e^{-\mu x} \bar{y}_{x x}^{T} \bar{Q}_{3} \Lambda_{1} \bar{y}_{x x}\right)\right]_{x=0}^{x=1} \\
& -\mu \int_{0}^{1} e^{-\mu x} \bar{y}_{x x}^{T} \bar{Q}_{3} \Lambda_{1} \bar{y}_{x x} d x .
\end{aligned}
$$

Using the boundary condition (49), it follows from (50)

$$
\begin{aligned}
\dot{V}_{12}= & -\left(e^{-\mu} y_{x x}^{T}(1) \bar{Q}_{3} \Lambda_{1} \bar{y}_{x x}(1)\right. \\
& \left.-\left(\Lambda_{1}^{-2} G_{r} \Lambda_{1}^{2} \bar{y}_{x x}(1)\right)^{T} \bar{Q}_{3} \Lambda_{1}\left(\Lambda_{1}^{-2} G_{r} \Lambda_{1}^{2} \bar{y}_{x x}(1)\right)\right) \\
& -\mu \int_{0}^{1} e^{-\mu x} \bar{y}_{x x}^{T} \bar{Q}_{3} \Lambda_{1} \bar{y}_{x x} d x .
\end{aligned}
$$

Developing and reorganizing (51) we get

$$
\begin{aligned}
\dot{V}_{12}= & -\bar{y}_{x x}^{T}(1) \Lambda_{1}^{2}\left(e^{-\mu} \Lambda_{1}^{-2} \bar{Q}_{3} \Lambda_{1}^{-1}\right. \\
& \left.-G_{r}^{T} \Lambda_{1}^{-2} \bar{Q}_{3} \Lambda_{1}^{-1} G_{r}\right) \Lambda_{1}^{2} \bar{y}_{x x}(1) \\
& -\mu \int_{0}^{1} e^{-\mu x} \bar{y}_{x x}^{T} \bar{Q}_{3} \Lambda_{1} \bar{y}_{x x} d x .
\end{aligned}
$$

By selecting $\bar{Q}_{3}=\Lambda_{1}^{2} \Delta_{1}^{2} \Lambda_{1}$, it is deduced from (52)

$$
\dot{V}_{12} \leqslant-\mu \int_{0}^{1} e^{-\mu x} \bar{y}_{x x}^{T} \bar{Q}_{3} \Lambda_{1} \bar{y}_{x x} d x .
$$

Combining (41), (47) and (53), $\dot{V}_{1}(\bar{y})$ follows

$$
\dot{V}_{1}(\bar{y}) \leqslant-\mu \lambda_{\min }\left(\Lambda_{1}\right) V_{1}(\bar{y}) .
$$

Therefore, there exists a positive value $C_{0}$ such that

$$
\|\bar{y}(., t)\|_{H^{2}}^{2} \leqslant C_{0} e^{-\mu \lambda_{\min }\left(\Lambda_{1}\right) t}\left\|\bar{y}^{0}\right\|_{H^{2}}^{2} .
$$

This concludes the proof of Lemma 2.

Let us state the proof of Theorem 2 .

Proof. Combining (26) in Lemma 1, (35) and (36) in Lemma 2 yields

$\dot{V}_{\epsilon}(\eta, \delta) \leqslant-\mu \int_{0}^{1} e^{-\mu x} \eta^{T} Q \Lambda_{1} \eta d x$

$-\left(\mu-\frac{\epsilon \kappa\left\|P\left(I_{m}-G_{22}\right)^{-1} G_{21} \Lambda_{1}\right\|}{\lambda_{\min }\left(P \Lambda_{2}\right)}\right) \int_{0}^{1} e^{-\mu x} \delta^{T} P \Lambda_{2} \delta d x$

$+\frac{3 \epsilon C^{0}\left\|P\left(I_{m}-G_{22}\right)^{-1} G_{21} \Lambda_{1}\right\|}{\kappa} e^{-\mu \lambda_{\min }\left(\Lambda_{1}\right) t}\left\|\bar{y}^{0}\right\|_{H^{2}}^{2}$. 
By choosing $\kappa=\frac{\mu \lambda_{\min }\left(P \Lambda_{2}\right)}{2 \epsilon\left\|P\left(I_{m}-G_{22}\right)^{-1} G_{21} \Lambda_{1}\right\|}$, we get

$\dot{V}_{\epsilon}(\eta, \delta) \leqslant-\frac{\mu \beta}{2} V_{\epsilon}(\eta, \delta)$

$+\frac{6 \epsilon^{2} C_{0}\left\|P\left(I_{m}-G_{22}\right)^{-1} G_{21} \Lambda_{1}\right\|^{2}}{\mu \lambda_{\min }\left(P \Lambda_{2}\right)} e^{-\mu \lambda_{\min }\left(\Lambda_{1}\right) t}\left\|\bar{y}^{0}\right\|_{H^{2}}^{2}$,

where $\beta=\min \left(\lambda_{\min }\left(\Lambda_{1}\right), \lambda_{\min }\left(\Lambda_{2}\right)\right)$.

With $\lambda_{\min }\left(\Lambda_{1}\right)>\frac{\beta}{2}$, it follows from $(56)$

$V_{\epsilon}(\eta, \delta) \leqslant e^{-\frac{\mu \beta}{2} t} V_{\epsilon}\left(\eta^{0}, \delta^{0}\right)$

$+\frac{6 \epsilon^{2} C_{0}\left\|P\left(I_{m}-G_{22}\right)^{-1} G_{21} \Lambda_{1}\right\|^{2}}{\mu^{2} \lambda_{\min }\left(P \Lambda_{2}\right)\left(\lambda_{\min }\left(\Lambda_{1}\right)-\frac{\beta}{2}\right)} e^{-\frac{\mu \beta}{2} t}\left\|\bar{y}^{0}\right\|_{H^{2}}^{2}$.

Note that

$$
\begin{aligned}
& e^{-\mu} \lambda_{\min }(Q)\|\eta\|_{L^{2}}^{2}+\epsilon e^{-\mu} \lambda_{\min }(P)\|\delta\|_{L^{2}}^{2} \\
\leqslant & V_{\epsilon}(\eta, \delta) \\
\leqslant & \|Q\|\|\eta\|_{L^{2}}^{2}+\epsilon\|P\|\|\delta\|_{L^{2}}^{2} .
\end{aligned}
$$

It follows that

$$
\begin{aligned}
\| \eta(., t) & \|_{L^{2}}^{2} \leqslant \frac{e^{\mu}}{\lambda_{\min }(Q)} V_{\epsilon}(\eta, \delta) \\
\leqslant & \frac{e^{\mu}}{\lambda_{\min }(Q)}\left(e^{-\frac{\mu \beta}{2} t} V_{\epsilon}\left(\eta^{0}, \delta^{0}\right)\right. \\
+ & \left.\frac{6 \epsilon^{2} C_{0}\left\|P\left(I_{m}-G_{22}\right)^{-1} G_{21} \Lambda_{1}\right\|^{2}}{\mu^{2} \lambda_{\min }\left(P \Lambda_{2}\right)\left(\lambda_{\min }\left(\Lambda_{1}\right)-\frac{\beta}{2}\right)} e^{-\frac{\mu \beta}{2} t}\left\|\bar{y}^{0}\right\|_{H^{2}}^{2}\right) \\
\leqslant & \frac{e^{\mu}}{\lambda_{\min }(Q)}\left(e^{-\frac{\mu \beta}{2} t}\left(\|Q\|\left\|\eta^{0}\right\|_{L^{2}}^{2}+\epsilon\|P\|\left\|\delta^{0}\right\|_{L^{2}}^{2}\right)\right. \\
+ & \left.\frac{6 \epsilon^{2} C_{0}\left\|P\left(I_{m}-G_{22}\right)^{-1} G_{21} \Lambda_{1}\right\|^{2}}{\mu^{2} \lambda_{\min }\left(P \Lambda_{2}\right)\left(\lambda_{\min }\left(\Lambda_{1}\right)-\frac{\beta}{2}\right)} e^{-\frac{\mu \beta}{2} t}\left\|\bar{y}^{0}\right\|_{H^{2}}^{2}\right)
\end{aligned}
$$

as $y^{0}=\bar{y}^{0}$, i.e. $\eta^{0}=0$, therefore

$$
\begin{aligned}
& \|\eta(., t)\|_{L^{2}}^{2} \leqslant \epsilon C_{a} e^{-\frac{\mu \beta}{2} t}\left\|\delta^{0}\right\|_{L^{2}}^{2} \\
& \quad+\frac{6 \epsilon^{2} C_{0} C_{a}\left\|P\left(I_{m}-G_{22}\right)^{-1} G_{21} \Lambda_{1}\right\|^{2}}{\mu^{2}\|P\| \lambda_{\min }\left(P \Lambda_{2}\right)\left(\lambda_{\min }\left(\Lambda_{1}\right)-\frac{\beta}{2}\right)} e^{-\frac{\mu \beta}{2} t}\left\|\bar{y}^{0}\right\|_{H^{2}}^{2}
\end{aligned}
$$

where $C_{a}$ is a given positive value. Thus we get the estimate

$$
\begin{aligned}
& \|y(., t)-\bar{y}(., t)\|_{L^{2}}^{2} \leqslant \epsilon C_{a} e^{-\frac{\mu \beta}{2} t}\left\|\delta^{0}\right\|_{L^{2}}^{2} \\
& \quad+\frac{6 \epsilon^{2} C_{0} C_{a}\left\|P\left(I_{m}-G_{22}\right)^{-1} G_{21} \Lambda_{1}\right\|^{2}}{\mu^{2}\|P\| \lambda_{\min }\left(P \Lambda_{2}\right)\left(\lambda_{\min }\left(\Lambda_{1}\right)-\frac{\beta}{2}\right)} e^{-\frac{\mu \beta}{2} t}\left\|\bar{y}^{0}\right\|_{H^{2}}^{2} .
\end{aligned}
$$

This proves (21).

Remarking the first term in the right hand side part in (55) is negative and taking the same $\kappa=$ $\frac{\mu \lambda_{\min }\left(P \Lambda_{2}\right)}{2 \epsilon\left\|P\left(I_{m}-G_{22}\right)^{-1} G_{21} \Lambda_{1}\right\|}$ yield

$$
\begin{aligned}
& \dot{V}_{\epsilon}(\eta, \delta) \leqslant-\frac{\mu}{2} e^{-\mu} \lambda_{\min }\left(P \Lambda_{2}\right)\|\delta(., t)\|_{L^{2}}^{2} \\
& +\frac{6 \epsilon^{2} C_{0}\left\|P\left(I_{m}-G_{22}\right)^{-1} G_{21} \Lambda_{1}\right\|^{2}}{\mu \lambda_{\min }\left(P \Lambda_{2}\right)} e^{-\mu \lambda_{\min }\left(\Lambda_{1}\right) t}\left\|\bar{y}^{0}\right\|_{H^{2}}^{2}
\end{aligned}
$$

Performing the time integration of both sides of (59) and using $\lim _{t \rightarrow+\infty} V_{\epsilon}(\eta, \delta)=0$, we get

$$
\begin{aligned}
0-V_{\epsilon}\left(\eta^{0}, \delta^{0}\right) & \leqslant-\frac{\mu}{2} e^{-\mu} \lambda_{\min }\left(P \Lambda_{2}\right) \int_{0}^{\infty}\|\delta(., t)\|_{L^{2}}^{2} d t \\
& +\frac{6 \epsilon^{2} C_{0}\left\|P\left(I_{m}-G_{22}\right)^{-1} G_{21} \Lambda_{1}\right\|^{2}}{\mu^{2} \lambda_{\min }\left(P \Lambda_{2}\right) \lambda_{\min }\left(\Lambda_{1}\right)}\left\|\bar{y}^{0}\right\|_{H^{2}}^{2}
\end{aligned}
$$

Reorganizing (60), we have

$$
\begin{aligned}
\int_{0}^{\infty}\|\delta(., t)\|_{L^{2}}^{2} d t \leqslant \frac{2 e^{\mu}}{\mu \lambda_{\min }\left(P \Lambda_{2}\right)}\left(V_{\epsilon}\left(\eta^{0}, \delta^{0}\right)\right. \\
\left.+\frac{6 \epsilon^{2} C_{0}\left\|P\left(I_{m}-G_{22}\right)^{-1} G_{21} \Lambda_{1}\right\|^{2}}{\mu^{2} \lambda_{\min }\left(P \Lambda_{2}\right) \lambda_{\min }\left(\Lambda_{1}\right)}\left\|\bar{y}^{0}\right\|_{H^{2}}^{2}\right) .
\end{aligned}
$$

Therefore with (58) and $\eta^{0}=0$, it follows

$$
\int_{0}^{\infty}\|\delta(., t)\|_{L^{2}}^{2} d t \leqslant \epsilon C_{b 1}\left\|\delta^{0}\right\|_{L^{2}}^{2}+\epsilon^{2} C_{b 2}\left\|\bar{y}^{0}\right\|_{H^{2}}^{2}
$$

where $C_{b 1}$ and $C_{b 2}$ are positive constants. This is equivalent to

$$
\begin{aligned}
& \int_{0}^{\infty}\left\|z(., t)-\left(I_{m}-G_{22}\right)^{-1} G_{21} \bar{y}(1, t)\right\|_{L^{2}}^{2} d t \\
\leqslant & \epsilon C_{b 1}\left\|\delta^{0}\right\|_{L^{2}}^{2}+\epsilon^{2} C_{b 2}\left\|\bar{y}^{0}\right\|_{H^{2}}^{2},
\end{aligned}
$$

and proves (22). This concludes the proof of Theorem 2 .

Corollary 1 There exists $C^{\prime \prime}>0$ such that if $z^{0}$ is the equilibrium point, that is $z^{0}=\left(I_{m}-G_{22}\right)^{-1} G_{21} \bar{y}^{0}(1)$, then for any $\bar{y}^{0} \in H^{2}(0,1)$ and for $t \geqslant 0$, the following estimate holds,

$$
\left\|z(., t)-\left(I_{m}-G_{22}\right)^{-1} G_{21} \bar{y}(1, t)\right\|_{L^{2}}^{2} \leqslant C^{\prime \prime} \epsilon e^{-\omega t} .
$$

Proof. The proof of this corollary is similar to that of Theorem 2. Due to (57) and (58), since $\eta^{0}=0$ and 
$\delta^{0}=0$, therefore

$$
\leqslant \epsilon \frac{6 C_{0} C_{b}\left\|P\left(I-G_{22}\right)^{-1} G_{21} \Lambda_{1}\right\|^{2}}{\mu^{2} \lambda_{\min }\left(P \Lambda_{2}\right)\left(\lambda_{\min }\left(\Lambda_{1}\right)-\frac{\beta}{2}\right)} e^{-\frac{\mu \beta}{2} t}\left\|\bar{y}^{0}\right\|_{H^{2}}^{2},
$$

where $C_{b}>0$. This proves (61).

\section{Application to a gas transport model}

\subsection{System description}

In this section, we consider a gas transport model which is an example of linear singularly perturbed system of conservation laws to illustrate the results of Sections 3 and 4 .

The gas dynamics through a tube with constant cross section, where all the friction losses and heat transfers are neglected, are usually modelled by the following Euler equations as considered in Winterbone (2000), Castillo et al. (2012).

$$
W_{t}+A W_{x}=0
$$

with $W=\left(\begin{array}{l}u \\ \rho \\ p\end{array}\right), A=\left(\begin{array}{rrr}u & 0 & \frac{1}{\rho} \\ \rho & u & 0 \\ a^{2} \rho & 0 & u\end{array}\right)$.

where

- $u=u(x, t)$ stands for the gas velocity at location $x$ in $[0,1]$ (we assume that the length of the tube equals 1 ) and at time $t$,

- $\rho=\rho(x, t)$ represents the gas density,

- $p=p(x, t)$ is the gas pressure,

- $a$ is sound speed in ideal gas.

The system (62) admits a steady-state $u^{*}, \rho^{*}, p^{*}$. The deviations of the state $u, \rho, p$ with respect to the steadystate are defined as

$$
\begin{aligned}
& \hat{u}=u-u^{*}, \\
& \hat{\rho}=\rho-\rho^{*}, \\
& \hat{p}=p-p^{*} .
\end{aligned}
$$

Then the linearization of system (62) at this equilibrium is

$$
\hat{W}_{t}+A^{*} \hat{W}_{x}=0,
$$

with $\hat{W}=\left(\begin{array}{c}\hat{u} \\ \hat{\rho} \\ \hat{p}\end{array}\right), A^{*}=\left(\begin{array}{ccc}u^{*} & 0 & \frac{1}{\rho^{*}} \\ \rho^{*} & u^{*} & 0 \\ a^{* 2} \rho^{*} & 0 & u^{*}\end{array}\right)$.

Let perform a change of coordinates for (63) to diagonalize $A^{*}$,

$$
S=L^{-1} \hat{W}, \Lambda=L^{-1} A^{*} L
$$

where $L=\left(\begin{array}{ccc}0 & 1 & 1 \\ 1 & -\frac{\rho^{*}}{a^{*}} & \frac{\rho^{*}}{a^{*}} \\ 0 & -a^{*} \rho^{*} & a^{*} \rho^{*}\end{array}\right)$.

We obtain the following diagonal hyperbolic system of conservation laws

$$
S_{t}+\Lambda S_{x}=0
$$

with $\Lambda=\left(\begin{array}{ccc}u^{*} & 0 & 0 \\ 0 & u^{*}-a^{*} & 0 \\ 0 & 0 & u^{*}+a^{*}\end{array}\right)$.

Assume that the propagation speed of gas is much slower than the sound speed, i.e. $u<<a$. By performing the change of spatial variable $S_{2}^{\prime}(1-x, t)=S_{2}(x, t)$, we may assume without loss of generality that the matrix $\Lambda>0$. A small positive value can be defined as $\epsilon=\frac{u^{*}}{a^{*}}$ and we use a new time scale $\tilde{t}=u^{*} t$, then (65) can be approximated by a linear singularly perturbed system of conservation laws

$$
\left(\begin{array}{l}
S_{1} \\
S_{2}^{\prime} \\
S_{3}
\end{array}\right)_{\tilde{t}}+\left(\begin{array}{ccc}
1 & 0 & 0 \\
0 & \frac{1}{\epsilon} & 0 \\
0 & 0 & \frac{1}{\epsilon}
\end{array}\right)\left(\begin{array}{c}
S_{1} \\
S_{2}^{\prime} \\
S_{3}
\end{array}\right)_{x}=0 .
$$

The boundary conditions needed to be imposed in system (66) can be expressed as follows

$$
\left(\begin{array}{l}
S_{1}(0, \widetilde{t}) \\
S_{2}^{\prime}(0, \widetilde{t}) \\
S_{3}(0, \widetilde{t})
\end{array}\right)=G\left(\begin{array}{l}
S_{1}(1, \widetilde{t}) \\
S_{2}^{\prime}(1, \widetilde{t}) \\
S_{3}(1, \widetilde{t})
\end{array}\right)
$$

where $G=\left(\begin{array}{lll}g_{11} & g_{12} & g_{13} \\ g_{21} & g_{22} & g_{23} \\ g_{31} & g_{32} & g_{33}\end{array}\right)$.

In system (66), $S_{1}$ stands for the slow dynamics and $S_{2}^{\prime}$, $S_{3}$ stand for the fast dynamics. Following the definitions of the two subsystems in Section 2, the reduced system of (66) and (67) is computed as follows

$$
\bar{S}_{1 \widetilde{t}}+\bar{S}_{1 x}=0
$$

with the boundary condition

$$
\bar{S}_{1}(0, \widetilde{t})=G_{r} \bar{S}_{1}(1, \widetilde{t}),
$$

where $G_{r}=g_{11}+\left(\begin{array}{ll}g_{12} & g_{13}\end{array}\right)\left(I_{2}-\left(\begin{array}{ll}g_{22} & g_{23} \\ g_{32} & g_{33}\end{array}\right)\right)^{-1}\left(\begin{array}{l}g_{21} \\ g_{31}\end{array}\right)$. The boundary-layer system is

$$
\left(\begin{array}{c}
\bar{S}_{2}^{\prime} \\
\bar{S}_{3}
\end{array}\right)_{\widetilde{\tau}}+\left(\begin{array}{c}
\bar{S}_{2}^{\prime} \\
\bar{S}_{3}
\end{array}\right)_{x}=0
$$


with the boundary conditions

$$
\left(\begin{array}{c}
\bar{S}_{2}^{\prime}(0, \widetilde{\tau}) \\
\bar{S}_{3}(0, \widetilde{\tau})
\end{array}\right)=G_{22}\left(\begin{array}{c}
\bar{S}_{2}^{\prime}(1, \widetilde{\tau}) \\
\bar{S}_{3}(1, \widetilde{\tau})
\end{array}\right)
$$

where $G_{22}=\left(\begin{array}{ll}g_{22} & g_{23} \\ g_{32} & g_{33}\end{array}\right), \widetilde{\tau}=\frac{\widetilde{t}}{\epsilon}$.

\subsection{Boundary conditions}

The plant is equipped with two fans located at each end of the tube, the rotation speed of each fan is seen as control action. The gas mass density at the output and the gas pressure at both ends of the tube are assumed to be measured. Precisely, we consider here the following boundary conditions for system (62):

1. The first boundary condition describes the operation of the inflow fan (see the fan specification map in Witrant et al. (2008)). It controls the input flow:

$$
u(0, \widetilde{t}) \alpha=g_{f} c_{0}(\widetilde{t})\left(p(0, \widetilde{t})-p_{i n}\right),
$$

where $\alpha$ is the constant cross section of the tube, $g_{f}$ is a constant coefficient, $c_{0}(\widetilde{t})$ denotes the rotation speed which is a control input, and $p_{i n}$ is a constant pressure before the inflow fan.

2 . The second boundary condition is given by the outflow fan which is used to control the output flow:

$$
u(1, \widetilde{t}) \alpha=g_{f} c_{1}(\widetilde{t})\left(p_{\text {out }}-p(1, \widetilde{t})\right)
$$

the control input is noted $c_{1}(\widetilde{t})$ and $p_{\text {out }}$ is a constant pressure behind the outflow fan.

3. The third boundary condition is a physical constraint, noting that the change of gas density through the input fan is small because of the pressure at the boundary $x=0$ is near atmospheric pressure, (see Castillo et al. (2012)):

$$
\rho(0, \widetilde{t})=\rho^{\prime}
$$

where $\rho^{\prime}$ is a constant value.

After the linearization of the above three boundary conditions, we have the following boundary conditions for system (63):

$$
\begin{aligned}
\hat{u}(0, \widetilde{t}) \alpha & =g_{f}\left[\hat{c}_{0}(\widetilde{t})\left(p^{*}-p_{\text {in }}\right)+c_{0}^{*} \hat{p}(0, \widetilde{t})\right], \\
\hat{u}(1, \widetilde{t}) \alpha & =g_{f}\left[\hat{c}_{1}(\widetilde{t})\left(p_{\text {out }}-p^{*}\right)-c_{1}^{*} \hat{p}(1, \widetilde{t})\right], \\
\hat{\rho}(0, \widetilde{t}) & =0,
\end{aligned}
$$

where $c_{0}^{*}, c_{1}^{*}$ are the constant control actions at the equilibrium $\left(u^{*}, \rho^{*}, p^{*}\right) . \hat{c}_{0}(\widetilde{t})=c_{0}(\widetilde{t})-c_{0}^{*}$ and $\hat{c}_{1}(\widetilde{t})=$ $c_{1}(\widetilde{t})-c_{1}^{*}$.

For a suitable choice of the control inputs $c_{0}(\widetilde{t})$ and $c_{1}(\widetilde{t})$, and for constant values $g_{21}, g_{23} \neq 1, g_{32} \neq 1$ in $\mathbb{R}$, the following conditions are equivalent to (75)-(77).

$$
\begin{aligned}
& S_{1}(0, \widetilde{t})=\frac{\rho^{*}\left(1-g_{32}\right)}{a^{*}} S_{2}^{\prime}(1, \widetilde{t}), \\
& S_{2}^{\prime}(0, \widetilde{t})=g_{21} S_{1}(1, \widetilde{t})+g_{23} S_{3}(1, \widetilde{t}), \\
& S_{3}(0, \widetilde{t})=g_{32} S_{2}^{\prime}(1, \widetilde{t}) .
\end{aligned}
$$

More precisely from (64) and (80), under the condition $g_{32}-1 \neq 0$, we can compute $\hat{u}(0, \widetilde{t})$ as a function of $\hat{p}(0, \widetilde{t})$,

$$
\hat{u}(0, \widetilde{t})=\frac{1+g_{32}}{a^{*} \rho^{*}\left(g_{32}-1\right)} \hat{p}(0, \widetilde{t}),
$$

and substituting (81) into (75), the control action at input of the tube is

$$
c_{0}(\widetilde{t})=c_{0}^{*}+\frac{\frac{\alpha\left(1+g_{32}\right)}{g_{f} a^{*} \rho^{*}\left(g_{32}-1\right)}-c_{0}^{*}}{p^{*}-p_{i n}} \hat{p}(0, \widetilde{t}) .
$$

Similarly, due to (64), (76) and (79), under the condition $1-g_{23} \neq 0$, the control action at output of the tube is given by

$$
\begin{aligned}
c_{1}(\widetilde{t})= & c_{1}^{*}+\frac{\frac{\alpha\left(a^{*}\left(1+g_{23}\right)-2 \rho^{*} g_{21}\right)}{g_{f} a^{* 2} \rho^{*}\left(1-g_{23}\right)}+c_{1}^{*}}{p_{\text {out }}-p^{*}} \hat{p}(1, \widetilde{t}) \\
& +\frac{\frac{2 \alpha g_{21}}{g_{f}\left(1-g_{23}\right)}}{p_{\text {out }}-p^{*}} \hat{\rho}(1, \widetilde{t}) .
\end{aligned}
$$

Since the outputs of (62) are the gas pressure at both ends of the tube and the gas mass density at the output, the control actions $c_{0}(\widetilde{t})$ in $(82)$ and $c_{1}(\widetilde{t})$ in $(83)$ are the feedback laws.

The boundary condition $G$ in (67) is written as follows

$$
G=\left(\begin{array}{ccc}
0 & \frac{\rho^{*}\left(1-g_{32}\right)}{a^{*}} & 0 \\
g_{21} & 0 & g_{23} \\
0 & g_{32} & 0
\end{array}\right)
$$

To ensure that the boundary conditions matrix $G$ satisfies $\rho_{1}(G)<1$, a straightforward choice for $\Delta$ is $\Delta=I_{3}$, then

$$
\Delta G \Delta^{-1}=\left(\begin{array}{ccc}
0 & \frac{\rho^{*}\left(1-g_{32}\right)}{a^{*}} & 0 \\
g_{21} & 0 & g_{23} \\
0 & g_{32} & 0
\end{array}\right) .
$$

Straightforward calculations show that $\left\|\Delta G \Delta^{-1}\right\|<1$ holds if and only if $g_{21}, g_{23}$ and $g_{32}$ can be selected as

$$
\begin{aligned}
g_{21}^{2}+g_{23}^{2} & <1, \\
\frac{\rho^{* 2}\left(1-g_{32}\right)^{2}}{a^{* 2}}+g_{32}^{2} & <1 .
\end{aligned}
$$




\section{$5.3 \quad$ Numerical solutions}

To numerically compute the solutions of (66) and (68), let us discretize them using a two-step variant of the Lax-Wendroff method which is presented in Shampine (2005b) and the solver on Matlab in Shampine (2005a). More precisely, we divide the space domain $[0,1]$ into 100 intervals of identical length, and 3 as final time. We choose a time-step $d t=0.9 \epsilon d x$ that satisfies the CFL condition for the stability and select the following initial functions:

$$
\begin{aligned}
S_{1}^{0} & =\bar{S}_{1}^{0}=\cos (4 \pi x)-1, \\
S_{2}^{\prime 0} & =\sin (5 \pi x), \\
S_{3}^{0} & =-\sin (5 \pi x) .
\end{aligned}
$$

The boundary conditions (84) are given by $g_{21}=0.1$, $g_{23}=0, g_{32}=0.2, a^{*}=340, \rho^{*}=1.2$. The condition $\rho_{1}(G)<1$ holds, thus Proposition 1 and Theorem 2 apply.

In the following figures and tables, $\eta, \delta_{1}$ and $\delta_{2}$ denote: $\cdot \eta=S_{1}-\bar{S}_{1}$,

$\cdot \delta_{1}=S_{2}^{\prime}-\frac{g_{21}}{1-g_{23} g_{32}} \bar{S}_{1}(1, \widetilde{t})$,

- $\delta_{2}=S_{3}-\frac{g_{21} g_{32}}{1-g_{23} g_{32}} \bar{S}_{1}(1, \widetilde{t})$.

Figure 1 shows the time evolution of the solution $\bar{S}_{1}$ for the reduced system (68)-(69). It is observed that $\bar{S}_{1}$ converges to the origin as time increases, as expected from Proposition 1. Time evolution of $\eta$ is shown in Figure 2. It is found that the error between $S_{1}$ in the singularly perturbed system (66)-(67) and $\bar{S}_{1}$ in the reduced system (68)-(69) is small and close to 0 as time increases. Figure 3 and Figure 4 represent the time evolutions of $\delta_{1}$ and $\delta_{2}$, which are the differences between $S_{2}^{\prime}, S_{3}$ and their equilibriums $\frac{g_{21}}{1-g_{23} g_{32}} \bar{S}_{1}(1, \widetilde{t}), \frac{g_{21} g_{32}}{1-g_{23} g_{32}} \bar{S}_{1}(1, \widetilde{t})$ respectively. The differences decrease to 0 as time increases.

Table 1 gives the evolution of square of $L^{2}$-norm of $\eta$ with the different values of $\epsilon$. It indicates that the difference between $S_{1}$ in (66)-(67) and $\bar{S}_{1}$ in (68)-(69) is near zero, and it decreases as $\epsilon$ decreases. Table 2 shows that the time integrals of square of $L^{2}$-norm of $\delta_{1}$ and $\delta_{2}$ decrease as $\epsilon$ decreases, and they are close to zero, as expected from Theorem 2 .

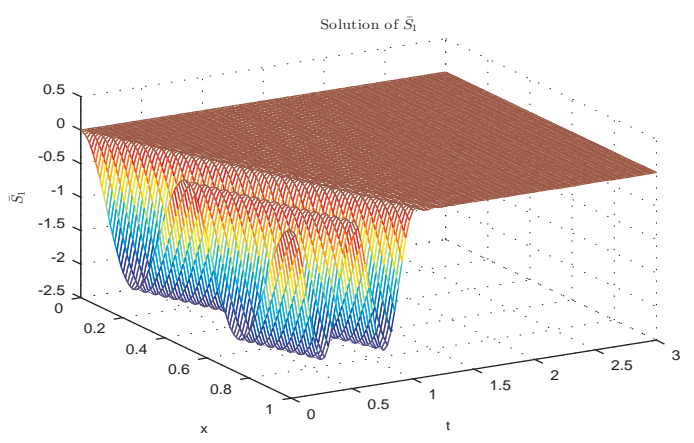

Fig. 1. Time evolution of the solution $\bar{S}_{1}$ in the reduced system (68)-(69).

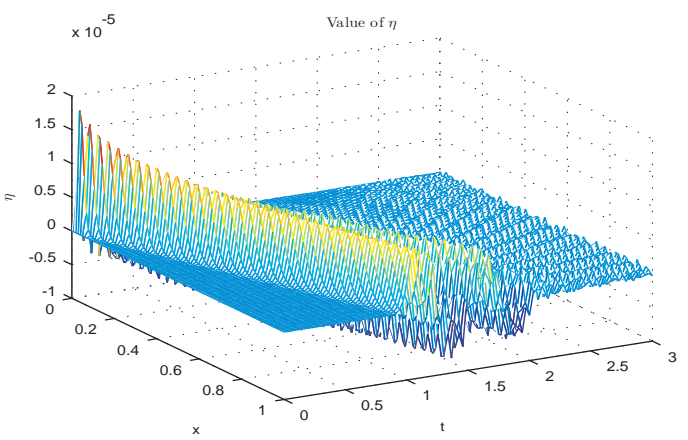

Fig. 2. Time evolution of $\eta$ which is difference between $S_{1}$ in the full system (66)-(67) and $\bar{S}_{1}$ in the reduced system (68)-(69).

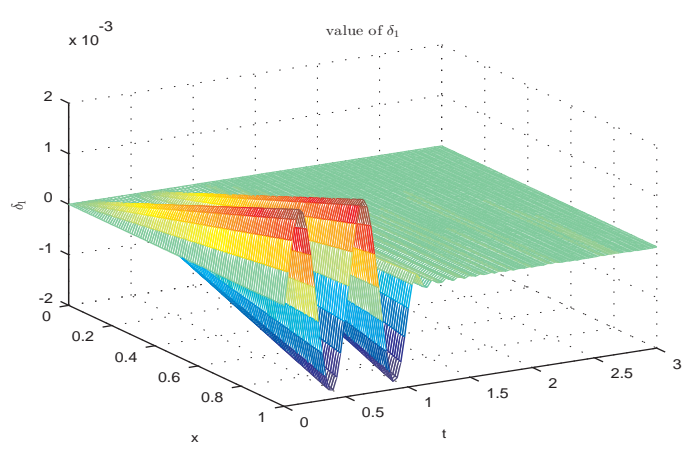

Fig. 3. Time evolution of $\delta_{1}$ which is the difference between $S_{2}^{\prime}$ and its equilibrium $\frac{g_{21}}{1-g_{23} g_{32}} \bar{S}_{1}(1, t)$.

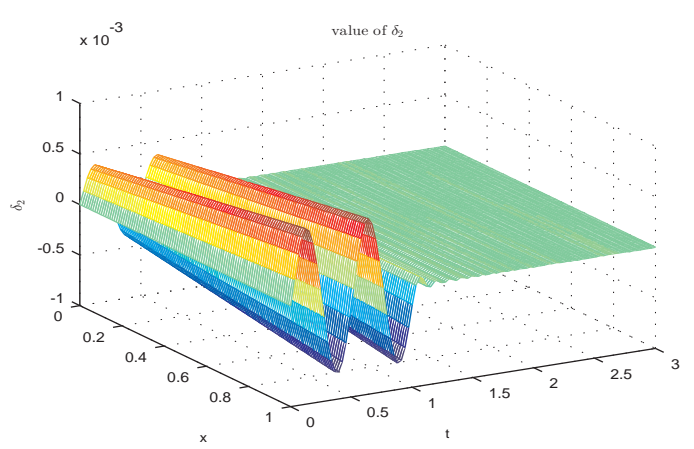

Fig. 4. Time evolution of $\delta_{2}$ which is the difference between $S_{3}$ and its equilibrium $\frac{g_{21} g_{32}}{1-g_{23} g_{32}} \bar{S}_{1}(1, t)$.

Table 1

Evolution of square of $L^{2}$-norm of $\eta$ for different $\epsilon$

\begin{tabular}{|c|c|c|c|}
\hline$\epsilon$ & 0.004 & 0.003 & 0.001 \\
\hline$\|\eta(., t=1.5)\|_{L^{2}}^{2}$ & $7.0 \times 10^{-11}$ & $3.1 \times 10^{-11}$ & $7.9 \times 10^{-12}$ \\
\hline
\end{tabular}

Let us define $\iota$, which is the difference of the solution of the slow dynamics between (66) and (65) with the same boundary conditions matrix (84). Table 3 shows the value of square of $L^{2}$-norm of $\iota$ for different $\epsilon$. It is 
Table 2

Evolutions of time integral of square of $L^{2}$-norms of $\delta_{1}$ and $\delta_{2}$ for different $\epsilon$

\begin{tabular}{|c|c|c|c|}
\hline$\epsilon$ & 0.004 & 0.003 & 0.001 \\
\hline $\int_{0}^{3}\left\|\delta_{1}\right\|_{L^{2}}^{2} d t$ & $5.2 \times 10^{-6}$ & $2.3 \times 10^{-6}$ & $5.8 \times 10^{-7}$ \\
\hline $\int_{0}^{3}\left\|\delta_{2} \mid\right\|_{L^{2}}^{2} d t$ & $1.5 \times 10^{-6}$ & $6.5 \times 10^{-7}$ & $1.6 \times 10^{-7}$ \\
\hline
\end{tabular}

observed that $\iota$ is small and close to zero. This motivates the approximation of (65) by (66).

Table 3

Evolution of square of $L^{2}$-norm of $\iota$ for different $\epsilon$

\begin{tabular}{|c|c|c|c|}
\hline$\epsilon$ & 0.004 & 0.003 & 0.001 \\
\hline$\|\iota(., t=1.5)\|_{L^{2}}^{2}$ & $1.3 \times 10^{-15}$ & $2.7 \times 10^{-16}$ & $1.7 \times 10^{-17}$ \\
\hline
\end{tabular}

\section{Conclusion}

It has been considered a linear singularly perturbed system of conservation laws. The reduced system and the boundary-layer system have been computed. In Proposition 1 it has been first explained how to apply the stability condition for the full singularly perturbed system, $\rho_{1}(G)<1$, to the two subsystems stability analysis. However, a counter example has been given to show that the stability of the two subsystems does not guarantee the stability of the overall system.

Then in Theorem 2 it has been shown that, under the stability condition $\rho_{1}(G)<1$, the solution of the linear singularly perturbed system of conservation laws can be approximated by the solution of the reduced system. This theorem has been applied to a gas transport setup which can be modelled by a singularly perturbed system by employing the fact that the propagation speed of gas is much slower than the sound speed.

This work leaves many open questions. It is natural to extend this work to systems of balance laws. Another interesting point is to consider some other physical applications, like open channels as considered in Dos Santos and Prieur (2008). The nonlinear case in Coron et al. (2008) will be also considered in the future works.

\section{References}

Castillo, F., Witrant, E., Dugard, L., 2012. Contrôle de Température dans un Flux de Poiseuille. IEEE Conférence Internationale Francophone d'Automatique, Grenoble, France.

Chow, J., 1978. Asymptotic stability of a class of nonlinear singularly perturbed systems. J.Franklin Inst. 305 (5), 275-281.

Chow, J., Kokotović, P., 1981. A two-stage LyapunovBellman feedback design of a class of nonlinear systems. IEEE Trans. Automat. Contr. 26 (3), 656-663.
Coron, J.-M., 2007. Control and nonlinearity. Vol. 136 of Mathematical Surveys and Monographs. Americam Mathematical Society.

Coron, J.-M., Bastin, G., d'Andréa-Novel, B., 2008. Dissipative boundary conditions for one-dimensional nonlinear hyperbolic systems. SIAM Journal on Control and Optimization 47 (3), 1460-1498.

Diagne, A., Bastin, G., Coron, J.-M., 2012. Lyapunov exponential stability of 1-D linear hyperbolic systems of balance laws. Automatica 48, 109-114.

Dos Santos, V., Prieur, C., 2008. Boundary control of open channels with numerical and expermental validations. IEEE Trans. Control Syst. Tech. 16 (6), 1252 1264.

Grujic, L., 1981. Uniform asymptotic stability of nonlinear singularly perturbed and large scale systems. Int. J. Contr. 33 (3), 481-504.

Habets, P., 1974. Stabilité asymptotique pour des problèmes de perturbations singulières. Centro Internazionalle Matematico Estimo.

Hale, J. K., Lunel, S. M. V., 1993. Introduction to Functional-Differential Equations. Springer-Verlag, New York.

Kadalbajoo, M., Patidar, K., 2003. Singularly perturbed problems in partial differential equations: a survey. Applied Mathematics and Computation 134, 371-429.

Khalil, H. K., 1996. Nonlinear systems. Prentice-Hall.

Kokotović, P., Khalil, H., O’Reilly, J., 1986. Singular pertrubation methods in control: analysis and design. Academic Press.

Li, T.-T., 1994. Global classical solutions for quasilinear hyperbolic systems. RAM:Research in Applied Mathematics. Masson, Paris 32.

Perrollaz, V., Rosier, L., 2014. Finite-time stabilization of $2 \times 2$ hyperbolic systems on tree-shaped networks. SIAM Journal on Control and Optimization 52 (1), 143-163.

Saberi, A., Khalil, H., 1984. Quadratic-type Lyapunov Functions for Singularly Perturbed Systems. IEEE Trans. Automat. Contr. AC-29 (6), 542-550.

Shampine, L., 2005a. Solving hyperbolic PDEs in Matlab. Appl. Numer. Anal. Comput. Math. 2, 346-358.

Shampine, L., 2005b. Two-step Lax-Friedrichs method. Applied Mathematics Letters 18, 1134-1136.

Smith, D., 1985. Singular-perturbation theory: an introduction with application. Cambridge University Press.

Tang, Y., Prieur, C., Girard, A., 2013. A new H² Lyapunov function for the stability of a singularly perturbed system of two conservation laws. IEEE Conference on Decision and Control, Florence, Italy, 30263031.

Tang, Y., Prieur, C., Girard, A., 2014. Boundary control synthesis for hyperbolic systems: a singular perturbation approach. IEEE Conference on Decision and Control, Los Angeles, California, USA.

Verhulst, F., 2007. Singular perturbation methods for slow-fast dynamics. Nonlinear Dynamics 50 (4), 747753 . 
Winterbone, D. E., 2000. Theory of Engine Manifold Design: Wave Action Methods for IC Engines. Society of Automotive Engineers. Inc.

Witrant, E., Johansson, K., the HynX team, 2008. Air flow modelling in deep wells: application to mining ventilation. IEEE Conference on Automation Science and Engineering, 845-850. 